\title{
Positively Curved Complete Noncompact Kähler Manifolds
}

\author{
Bing-Long Chen , Xi-Ping Zhu \\ Department of Mathematics, Zhongshan University, \\ Guangzhou 510275, P. R. China, and \\ The Institute of Mathematical Sciences, The Chinese University of Hong \\ Kong, Hong Kong
}

\begin{abstract}
In this paper we give a partial affirmative answer to a conjecture of Greene$\mathrm{Wu}$ and Yau. We prove that a complete noncompact Kähler surface with positive and bounded sectional curvature and with finite analytic Chern number $c_{1}(M)^{2}$ is biholomorphic to $\mathbf{C}^{2}$.
\end{abstract}

\section{$\S 1$. Introduction}

The celebrated theorem of Cheeger-Gromoll-Meyer [3], [10] states that a complete noncompact Riemannian manifold with positive sectional curvature is diffeomorphic to the Euclidean space. It is well-known that there is a vast variety of biholomorphically distinct complex structures on $\mathbf{R}^{2 n}$ for $n>1$ ( see [2], [7] ). To understand the relationship between the Riemannian structure and the complex structure on complete noncompact manifolds, we restrict attention to Kähler manifolds which has the effect of insuring a closer relationship between these two structures. In [8], Greene and Wu proved that a complete noncompact Kähler manifold with positive sectional curvature is Stein. This fact thus motivated the following conjecture: 
Conjecture ( Greene-Wu 9] and Yau [27 ) A complete noncompact Kähler manifold of positive sectional curvature is biholomorphic to a complex Euclidean space.

In [14, among other things, Mok gave the first partial affirmative answer to the conjecture for complex two-dimensional manifolds with maximal volume growth.

Theorem ( Mok 14 ) Suppose $M$ is a complete noncompact Kähler manifold of complex dimension $n=2$. Suppose also $M$ has positive sectional curvature and satisfies

$$
\begin{aligned}
& \text { (i) } \quad 0<\text { scalar curvature } \leq \frac{C}{d^{2}\left(x_{0}, x\right)}, \\
& \text { (ii) } \quad \text { olume }\left(B\left(x_{0}, r\right)\right) \geq c r^{2 n}, \quad 0 \leq r<+\infty,
\end{aligned}
$$

where $B\left(x_{0}, r\right)$ and $d\left(x_{0}, x\right)$ denote respectively geodesic balls and geodesic distances, c, C are some positive constants. Then $M$ is biholomorphic to $\mathbf{C}^{2}$.

Denote by Ric the Ricci curvature form of $M$. As remarked in Mok [16], the conditions $(i)$ and $(i i)$ imply that the integral $\int_{M} R i c^{n}$, the analytic Chern number $c_{1}(M)^{n}$, is finite.

In his paper [26], To gave a generalization of the above result to nonmaximal volume growth manifolds. More precisely, it was proved in 26] that if $M$ is a complete noncompact Kähler manifold of positive sectional curvature with complex dimension $n=2$ and suppose for some base point $x_{0} \in M$ that there exist positive constants $C_{1}, C_{2}$ and $p$ such that 


$$
\begin{aligned}
& (i)^{\prime} \quad 0<\text { scalar curvature } \leq \frac{C_{1}}{d^{p}\left(x_{0}, x\right)}, \\
& (\text { ii })^{\prime} \quad \int_{\text {B( } \left.x_{0}, r\right)} \frac{1}{\left(1+d\left(x_{0}, x\right)\right)^{n p}} d x \leq C_{2} \log (r+2), \quad 0 \leq r<+\infty \\
& \quad \int_{M}^{R i c^{n}<+\infty,}
\end{aligned}
$$

then $M$ is biholomorphic to $\mathbf{C}^{2}$. Moreover in case of $p \geq 2$, the result is valid without assuming condition $(i i)^{\prime}$. Also from Yau's theorem (cf. [20]) that complete noncompact manifolds with nonnegative Ricci curvature have at least linear volume growth we see that the positive constant $p$ need to be assumed not less that $\frac{1}{n}$.

It is likely that the assumption ( Kähler surfaces with positive sectional curvature. The reason is that on a complete noncompact real four-manifold with positive sectional curvature we have the generalized Cohn-Vossen inequality

$$
\int_{M} \Theta \leq \chi\left(\mathbf{R}^{4}\right)<+\infty
$$

where $\Theta$ is the Gauss-Bonnet-Chern integrand. It is well-known that the integrand $\Theta$ is positive and it seems that the exterior product $\operatorname{Ric}^{2}$ is more or less comparable with the Gauss-Bonnet-Chern integrand $\Theta$. Meanwhile in views of Demailly's holomorphic Morse inequality [6] and the $L^{2}$-Riemann-Roch inequality in Nadel-Tsuji 18 (see also Tian[25]), the assumption (iii) is a natural condition for a complete Kähler manifold to be a quasi-projective manifold. However the assumptions on the curvature decay and the volume growth are more problemtic since they demand the geometry of the Kähler manifold at infinity to be somewhat uniform. The main purpose of this paper is to show that the assumption ( iii) alone is sufficient to guarantee that the Kähler surface is biholomorphic to $\mathbf{C}^{2}$. 
Main Theorem Let $M$ be a complex $n$-dimensional complete noncompact Kähler manifold with positive and bounded sectional curvature. Suppose that

$$
\int_{M} R i c^{n}<+\infty .
$$

Then $M$ is biholomorphic to a quasi-projective variety, and in case of dimenion $n=2, M$ is biholomorphic to $\mathbf{C}^{2}$.

We remark that there is a more ambitious conjecture due to Yau [27, [28], i.e., the question is to demonstrate that every complete noncompact Kähler manifold with positive holomorphic bisectional curvature is biholomorphic to the complex Euclidean space. But for such a Kähler manifold with positive holomorphic bisectional curvature, one doesn't even know whether the manifold is simply connected. Moreover it is also unknown whether the Kähler manifold is Stein, which is a conjecture of Siu [23]. In the companion paper 团, the authors and S. H. Tang gave a partial affirmative answer to the above Yau's conjecture. We proved that given a complete noncompact complex two-dimensional Kähler manifold $M$ of positive and bounded holomorphic bisectional curvature, suppose its geodesic balls have maximal volume and its scalar curvature decays to zero at infinity in the average sense, then $M$ is biholomorphic to $\mathbf{C}^{2}$.

The basic idea to approach these conjectures is to compactify the manifold $M$ as a quasi-projective variety. Siu, Yau [24] and Mok [14] initiated this program by first using the $L^{2}$-method of Andreotti-Vessentini and Hörmander to establish a Siegel's theorem for a field of meromorphic functions and then desingularizing the "birational" map associated to the Siegel theorem. The crux is how to choose a subfield of meromorphic functions with suitable estimates for the desingularization. In [14] Mok solved the Poincaré-Lelong equation to show that there is plenty of holomorphic functions of polynomial growth and that the subfield of meromorphic functions arising from quotients of holomor- 
phic functions of polynomial growth has the desirable estimates. In [4] the Ricci flow was used to understand the topology of the manifold and to deduce that this field generated by holomorphic functions of polynomial growth still has the desirable estimates. In [16] and [26], the quotient fields were defined from holomorphic plurianticanonical sections with polynomial growth or satisfying certain integrability conditions associated to the assumptions on the curvature decay and the volume growth respectively.

Note that one can construct $L^{2}$ holomorphic sections of the plurianticanonical system $\left\{K^{-q}, q>0\right\}$ without any assumption on the volume growth and the curvature decay. We observe in the present paper that the space of $L^{2}$ sections forms a graded algebra. Moreover by using some techniques and estimates developed from the Ricci flow, we are able to derive desirable Bézout estimates for the intersections of the zero divisors of holomorphic $L^{2}$ sections. Based on these estimates we can bound the Gauss-Bonnet integrals of curves which are the intersection of zero divisors of $L^{2}$ holomorphic plurianticanonical sections. The main theorem will then be proved in the following way: we first use the Bézout estimates to obtain a Siegel type theorem for the field of meromorphic functions of $M$ arising from the quotients of the $L^{2}$ sections; we then get a "meromorphic" map of $M$ into a projective algebraic variety; we next use the bounds on the Gauss-Bonnet integrals of the "algebraic" curves to show that the meromorphic map is almost surjective; finally we desingularize the map into a biholomorphism from $M$ to a quasi-projective variety.

The composition of this paper is as follows. In Section 2 we collect some basic results and prove the space of $L^{2}$ holomorphic plurianticanonical sections forms a $\mathbf{Z}^{+}$-graded algebra. In Section 3 we derive the Bézout estimates and obtain a gradient estimate by using the Ricci flow, and then we bound the Gauss-Bonnet integrals of the curves cut by $L^{2}$ holomorphic plurianticanonical sections. Section 4 is devoted to the proof of the main theorem. 
The authors are grateful to Professor S. T. Yau for his interest and encouragement. The second author would like to thank Professor G. Tian for helpful discussion. This work was partially supported by The IMS of The Chinese University of Hong Kong and the Foundation for Outstanding Young Scholars of China.

\section{$\S 2 . L^{2}$ holomorphic plurianticanonical sections}

First of all, let us recall the standard $L^{2}$-estimates of $\bar{\partial}$ of AndreottiVesentini [1] and Hörmander [11] in the case of Hermitian holomorphic line bundles over Kähler manifolds.

Theorem 2.1 (Andreotti-Vesentini [1], Hörmander [11] )

Let $(M, \omega)$ be a complete Kähler manifold equipped with a Kähler form $\omega$. Let $L$ be a Hermitian holomorphic line bundle on $M$ and denote by $C(L)$ the curvature form of $L$. Let $\varphi$ be a smooth function and $c(x)$ be a positive continuous functions on $M$ such that

$$
\sqrt{-1} \partial \bar{\partial} \varphi+C(L)+R i c \geq c(x) \omega
$$

Suppose $f$ is a $\bar{\partial}$-closed smooth $L$-valued $(0,1)$ form such that

$$
\int_{M} \frac{\|f\|^{2}}{c} e^{-\varphi}<+\infty
$$

Then the equation $\bar{\partial} u=f$ has a smooth solution with the following estimate

$$
\int_{M}\|u\|^{2} e^{-\varphi} \leq \int_{M} \frac{\|f\|^{2}}{c} e^{-\varphi} .
$$

We will need the following sub-mean-value inequality which can be found in [13]. Actually the sub-mean-value inequality can be obtained directly by 
using the Green formula and the standard estimate of the Green function on a Riemannian manifold with nonnegative Ricci curvature.

Lemma 2.2 Let $M$ be a complete Riemannian manifold with nonnegative Ricci curvature. Suppose $f$ is a nonnegative smooth subharmonic function. Then there exists a constant $C$, depending only on the dimension, such that for any $x_{0} \in M$, we have

$$
f\left(x_{0}\right) \leq C \cdot \frac{1}{\operatorname{Vol}\left(B\left(x_{0}, a\right)\right)} \int_{B\left(x_{0}, a\right)} f(x),
$$

for all $a>0$.

Now let $M$ be a complete Kähler manifold of complex dimension $n$ with positive Ricci curvature. Denote by $K$ the canonical line bundle and $\Gamma^{2}\left(M, K^{-q}\right)$ the space of square-integrable holomorphic sections of the plurianticanonical line bundle $K^{-q}$. Here $q$ is a positive integer. Fix a point $x \in M$, suppose $\left\{z_{1}, \cdots, z_{n}\right\}$ is a holomorphic coordinate system at $x$ with $z_{1}(x)=\cdots=$ $z_{n}(x)=0$. Let $\left\{s_{0}^{\prime}, \cdots, s_{n}^{\prime}\right\}$ be a system of local holomorphic sections of $K^{-q}$ at $x$ with $s_{0}^{\prime}(x) \neq 0, s_{1}^{\prime}(x)=\cdots=s_{n}^{\prime}(x)=0$ and $d\left(s_{i}^{\prime} / s_{0}^{\prime}\right)=d z_{i}, \quad(i=1, \cdots, n)$, near $x$. Without loss of generality, we may assume $\left\{\sum_{i=1}^{n}\left|z_{i}\right|^{2}<1\right\}$ be a holomorphic coordinate ball contained in $M$. Let $\rho$ be a smooth cut-off function on $M$ satisfying $\rho \equiv 1$ on $B\left(0, \frac{1}{2}\right)$ and $\rho \equiv 0$ outside $B(0,1)$. Since the Ricci curvature is strictly positive everywhere, one can find a positive integer $q$ such that

$$
(q+1) \operatorname{Ric}+(n+1) \sqrt{-1} \partial \bar{\partial}\left(\rho \log \sum_{i=1}^{n}\left|z_{i}\right|^{2}\right)>0
$$

Choose $L=K^{-q}, \varphi=(n+1) \rho \log \sum_{i=1}^{n}\left|z_{i}\right|^{2}$. By applying Theorem 2.1, we know that there exist global holomorphic sections $u_{0}, u_{1}, \cdots, u_{n}$ of $K^{-q}$ such that

$$
\bar{\partial} u_{i}=\bar{\partial}\left(\rho s_{i}^{\prime}\right)
$$


and

$$
\int_{M}\left\|u_{i}\right\|^{2} e^{-(n+1) \rho \log \sum_{i=1}^{n}\left|z_{i}\right|^{2}}<+\infty
$$

for $i=0,1, \cdots, n$.

Set

$$
s_{i}=\rho s_{i}^{\prime}-u_{i}, \quad i=0,1, \cdots, n
$$

Then each $s_{i} \in \Gamma^{2}\left(M, K^{-q}\right)$ and $s_{0}(x) \neq 0, d\left(s_{i} / s_{0}\right)=d z_{i}(i=1, \cdots, n)$ near $x$. This says that the meromorphic functions $s_{1} / s_{0}, \cdots, s_{n} / s_{0}$ give a local holomorphic coordinate system at $x$.

Similarly for arbitrary two points $x$ and $y$ in $M$, one can choose $s_{0}, s_{1} \in$ $\Gamma^{2}\left(M, K^{-q}\right)$ with $s_{0}(x) \neq 0, s_{0}(y) \neq 0, s_{1}(x) \neq 0$, and $s_{1}(y)=0$ so that the meromorphic function $f=s_{1} / s_{0}$ is holomorphic at $x$ and $y$ and satisfies $f(x) \neq 0$ and $f(y)=0$.

Hence we have showed the space $\bigcup_{q>0} \Gamma^{2}\left(M, K^{-q}\right)$ gives local holomorphic coordinates and separates points on $M$. Furthermore, we will show that $\bigcup_{q>0} \Gamma^{2}\left(M, K^{-q}\right)$ forms a graded algebra. More precisely, we have

Proposition 2.3 Suppose $M$ has positive and bounded sectional curvature. Then the space $\bigcup_{q>0} \Gamma^{2}\left(M, K^{-q}\right)$ forms a $\mathbf{Z}^{+}$-graded algebra. Moreover if $s \in \bigcup_{q>0} \Gamma^{2}\left(M, K^{-q}\right)$, then $\|s\|$ is bounded and $\|\nabla s\|$ is square-integrable.

Proof. From the standard Bochner-Kodaira formula,

$$
\triangle\|s\|^{2}=\|\nabla s\|^{2}-q R\|s\|^{2}
$$

where $R$ is the scalar curvature of $M$.

Suppose that the sectional curvature is bounded from above by a positive constant $K_{0}$. It is easy to see from (2.1)

$$
\triangle\|s\|^{2} \geq-q n^{2} K_{0}\|s\|^{2}
$$


Let $G=e^{\sqrt{q n^{2} K_{0}} \tau}\|s\|^{2}$ be a function defined on the product manifold $\widetilde{M}=$ $M \times \mathbf{R}$, equipped with the product metric. Let $\widetilde{\triangle}=\triangle+\frac{\partial^{2}}{\partial \tau^{2}}$ be the Laplacian operator. It is clear that $\widetilde{M}$ has nonnegative Ricci curvature and we have

$$
\widetilde{\triangle} G \geq 0
$$

Then by Lemma 2.2 and the standard volume comparison we have for any $x_{0} \in M$,

$$
\|s\|^{2}\left(x_{0}\right) \leq C \cdot \frac{e^{\sqrt{q n^{2} K_{0}} \cdot a}-e^{-\sqrt{q n^{2} K_{0}} \cdot a}}{\sqrt{q n^{2} K_{0}} \cdot a} \cdot \frac{1}{\operatorname{Vol}\left(B\left(x_{0}, a\right)\right)} \int_{B\left(x_{0}, a\right)}\|s\|^{2},
$$

for all $a>0$. Since $M$ has positive sectional curvature, it is well-known from Gromoll and Meyer [10 that the injectivity radius of $M$ at $x_{0}$ satisfies the following estimate

$$
\operatorname{inj}\left(M, x_{0}\right) \geq \frac{\pi}{\sqrt{K_{0}}} .
$$

In particular we have the volume estimate

$$
\operatorname{Vol}\left(B\left(x_{0}, \frac{\pi}{2 \sqrt{K_{0}}}\right)\right) \geq C\left(\frac{\pi}{2 \sqrt{K_{0}}}\right)^{2 n}
$$

for some positive constant $C$ depending only on the dimension. So letting $a=\frac{\pi}{2 \sqrt{K_{0}}}$ in (2.2) we conclude

$$
\sup _{x_{0} \in M}\|s\|^{2}\left(x_{0}\right) \leq C \cdot \frac{e^{\sqrt{\frac{q n^{2}}{4}} \cdot \pi}-e^{-\sqrt{\frac{q n^{2}}{4}} \cdot \pi}}{\sqrt{\frac{q n^{2}}{4}} \cdot \pi} \cdot\left(\frac{2 \sqrt{K_{0}}}{\pi}\right)^{2 n} \int_{M}\|s\|^{2}<+\infty .
$$

This shows that $\|s\|^{2}$ is bounded on $M$. Thus the space $\bigcup_{q>0} \Gamma^{2}\left(M, K^{-q}\right)$ forms a $\mathbf{Z}^{+}$-graded algebra under standard addition and multiplication over the complex numbers $\mathbf{C}$.

We now consider the $L^{2}$ estimate for the gradient of a holomorphic section $s \in \Gamma^{2}\left(M, K^{-q}\right)$. Suppose $r$ is the distance function from a fixed point $x_{0}$ on 
M. It follows from (2.1) that for any $a>0$,

$$
\begin{aligned}
\int_{B\left(x_{0}, a\right)}\|\nabla s\|^{2}\left(1-\frac{r}{a}\right)^{2} & =\int_{B\left(x_{0}, a\right)} \Delta\|s\|^{2}\left(1-\frac{r}{a}\right)^{2}+\int_{B\left(x_{0}, a\right)} q R\|s\|^{2}\left(1-\frac{r}{a}\right)^{2} \\
& \leq \frac{4}{a} \int_{B\left(x_{0}, a\right)}\|\nabla s\| \cdot\|s\| \cdot\left(1-\frac{r}{a}\right)+q n^{2} K_{0} \int\|s\|_{M}^{2} \\
& \leq \frac{1}{2} \int_{B\left(x_{0}, a\right)}\|\nabla s\|^{2}\left(1-\frac{r}{a}\right)^{2}+\left(\frac{8}{a^{2}}+q n^{2} K_{0}\right) \iint_{M}\|s\|^{2} .
\end{aligned}
$$

Letting $a \rightarrow+\infty$, we get

$$
\int_{M}\|\nabla s\|^{2} \leq 2 q n^{2} K_{0} \int_{M}\|s\|^{2}<+\infty .
$$

\section{$\S 3$. Bézout estimates and gradient estimate}

Let us first recall a cut-off function constructed in the book of Schoen and Yau 20] ( see Theorem 1.4.2 in 20]).

Lemma 3.1 ( Schoen-Yau [20], see also Shi [21])

Suppose $M$ is a complete Riemannian manifold with nonnegative and bounded sectional curvature. Then for any $x_{0} \in M, a>0$, there exists a positive function $\varphi_{a}(x) \in C^{\infty}(M)$ such that

( $\alpha) \quad \exp \left(-C\left(1+\frac{d\left(x, x_{0}\right)}{a}\right)\right) \leq \varphi_{a}(x) \leq \exp \left(-\left(1+\frac{d\left(x, x_{0}\right)}{a}\right)\right)$,

( $\beta) \quad\left|\nabla \varphi_{a}(x)\right| \leq \frac{C}{a} \cdot \varphi_{a}(x)$,

$(\gamma) \quad\left|\nabla_{i} \nabla_{j} \varphi_{a}(x)\right| \leq \frac{C}{a^{2}} \cdot \varphi_{a}(x)$,

for all $x \in M$, where $C$ is a positive constant depending only on the dimension. 
We now state and prove the first Bézout estimate for $\bigcup_{q>0} \Gamma^{2}\left(M, K^{-q}\right)$.

Proposition 3.2 Let $(M, \omega)$ be assumed as in the Main Theorem. Let $q_{1}, \cdots, q_{k}$ be positive integers and let $s_{i} \in \Gamma^{2}\left(M, K^{-q_{i}}\right), i=1,2, \cdots, k$. For a sequence $\varepsilon_{i}>0, i=1, \cdots, k$, define

$$
\zeta_{\varepsilon_{i}}\left(s_{i}\right)=\sqrt{-1} \partial \bar{\partial} \log \left(\left\|s_{i}\right\|^{2}+\varepsilon_{i}^{2}\right)+q_{i} \operatorname{Ric}, \quad i=1, \cdots, k
$$

Then

$$
\int_{M} \zeta_{\varepsilon_{1}}\left(s_{1}\right) \wedge \cdots \wedge \zeta_{\varepsilon_{k}}\left(s_{k}\right) \wedge R i c^{n-k} \leq C q_{1} \cdots q_{k} \int_{M} \operatorname{Ric}^{n}<+\infty
$$

where $C$ is a positive constant depending only on $n$.

Proof. We first consider the case $k=1$. For sufficiently small $\delta>0$ and $a \geq 1$, we know $\left\{\varphi_{a}>\delta\right\} \subset M$ is a compact domain with boundary $\partial\left\{\varphi_{a}>\delta\right\}=\left\{\varphi_{a}=\delta\right\}$. The Poincaré-Lelong equation gives, in the sense of $(1,1)$ currents,

$$
\sqrt{-1} \partial \bar{\partial} \log \|s\|^{2}=[s=0]-q \operatorname{Ric}
$$

where, by abuse of notation, $[s=0]$ also denotes the $(1,1)$ current defined by the divisor $[s=0]$ counting multiplicity. From the Poincaré-Lelong equation and the positivity of plurianticanonical line bundles we see from an easy computation that each $\zeta_{\varepsilon_{i}}\left(s_{i}\right)$ is a closed, nonnegative (1.1) form on $M$. By Lemma 3.1 , we have

$$
\begin{aligned}
& \int_{\left\{\varphi_{a}>\delta\right\}}\left(\varphi_{a}-\delta\right)^{2} \zeta_{\varepsilon}(s) \wedge \operatorname{Ric}^{n-1} \\
= & \int_{\left\{\varphi_{a}>\delta\right\}}\left(\varphi_{a}-\delta\right)^{2} \sqrt{-1} \partial \bar{\partial} \log \left(\|s\|^{2}+\varepsilon^{2}\right) \wedge R i c^{n-1}+\int_{\left\{\varphi_{a}>\delta\right\}} q\left(\varphi_{a}-\delta\right)^{2} \operatorname{Ric}^{n} \\
\leq & \frac{C}{a^{2}} \int_{\left\{\varphi_{a}>\delta\right\}} \log \left(1+\frac{\|s\|^{2}}{\varepsilon^{2}}\right) \omega \wedge R i c^{n-1}+q \int_{\left\{\varphi_{a}>\delta\right\}} \operatorname{Ric}^{n} \\
\leq & \frac{C K_{0}^{n-1}}{a^{2} \varepsilon^{2}} \int_{M}\|s\|^{2}+q \int_{M} \operatorname{Ric}^{n}
\end{aligned}
$$


where $K_{0}$ is the upper bound of the sectional curvature of $M$. Here and below we denote by $C$ various positive constants depending only on $n$. Then letting $\delta \rightarrow 0$ and $a \rightarrow+\infty$ we deduce that

$$
\int_{M} \zeta_{\varepsilon}(s) \wedge R i c^{n-1} \leq C q \int_{M} R i c^{n} .
$$

We next consider the case $k=2$. By integrating by parts, we get

$$
\begin{aligned}
& \int_{\left\{\varphi_{a}>\delta\right\}}\left(\varphi_{a}-\delta\right)^{3} \zeta_{\varepsilon_{1}}\left(s_{1}\right) \wedge \zeta_{\varepsilon_{2}}\left(s_{2}\right) \wedge R i c^{n-2} \\
\leq & \int_{\left\{\varphi_{a}>\delta\right\}}\left(\varphi_{a}-\delta\right)^{3} \sqrt{-1} \partial \bar{\partial} \log \left(\left\|s_{2}\right\|^{2}+\varepsilon_{2}^{2}\right) \wedge \zeta_{\varepsilon_{1}}\left(s_{1}\right) \wedge R i c^{n-2} \\
& \quad+\int_{\left\{\varphi_{a}>\delta\right\}} q_{2} \zeta_{\varepsilon_{1}}\left(s_{1}\right) \wedge \operatorname{Ric}^{n-1} \\
= & \int_{\left\{\varphi_{a}>\delta\right\}} \log \left(1+\frac{\left\|s_{2}\right\|^{2}}{\varepsilon_{2}^{2}}\right) \cdot \sqrt{-1} \partial \bar{\partial}\left(\left(\varphi_{a}-\delta\right)^{3}\right) \wedge \zeta_{\varepsilon_{1}}\left(s_{1}\right) \wedge \operatorname{Ric}^{n-2} \\
& \quad+\int_{\left\{\varphi_{a}>\delta\right\}} q_{2} \zeta_{\varepsilon_{1}}\left(s_{1}\right) \wedge R i c^{n-1} .
\end{aligned}
$$

The second term of the RHS of (3.2) is bounded from above by $C q_{1} q_{2} \int_{M} R i c^{n}$ by the previous estimate for the case $k=1$. To get the bound on the first term of RHS of (3.2), we now derive an integral estimate for the gradient. Recall that for each $i$,

$$
\zeta_{\varepsilon_{i}}\left(s_{i}\right)=\sqrt{-1} \partial \bar{\partial} \log \left(\left\|s_{i}\right\|^{2}+\varepsilon_{i}^{2}\right)+q_{i} R i c \geq 0 .
$$

Thus we have

$$
\int_{\left\{\varphi_{a}>\delta\right\}}\left(\varphi_{a}-\delta\right)^{2} \log \left(1+\frac{\left\|s_{i}\right\|^{2}}{\varepsilon_{i}^{2}}\right)\left(\sqrt{-1} \partial \bar{\partial} \log \left(\left\|s_{i}\right\|^{2}+\varepsilon_{i}^{2}\right)+q_{i} R i c\right) \wedge \omega^{n-1} \geq 0 .
$$

Integrating by parts and using Lemma 3.1, we get

$$
\begin{gathered}
\int_{\left\{\varphi_{a}>\delta\right\}}\left(\varphi_{a}-\delta\right)^{2} \sqrt{-1} \partial \log \left(1+\frac{\left\|s_{i}\right\|^{2}}{\varepsilon_{i}^{2}}\right) \wedge \bar{\partial} \log \left(1+\frac{\left\|s_{i}\right\|^{2}}{\varepsilon_{i}^{2}}\right) \wedge \omega^{n-1} \\
\leq \int_{\left\{\varphi_{a}>\delta\right\}} \frac{C}{a}\left(\varphi_{a}-\delta\right) \log \left(1+\frac{\left\|s_{i}\right\|^{2}}{\varepsilon_{i}^{2}}\right)\left\|\nabla \log \left(1+\frac{\left\|s_{i}\right\|^{2}}{\varepsilon_{i}^{2}}\right)\right\| \omega^{n} \\
+\int_{\left\{\varphi_{a}>\delta\right\}} q_{i}\left(\varphi_{a}-\delta\right)^{2} \log \left(1+\frac{\left\|s_{i}\right\|^{2}}{\varepsilon_{i}^{2}}\right) \operatorname{Ric} \wedge \omega^{n-1} .
\end{gathered}
$$


Recall from Proposition 2.3 that each $s_{i}$ is bounded on $M$. By using CauchySchwarz inequality, we get

$$
\begin{aligned}
& \int_{\left\{\varphi_{a}>\delta\right\}}\left(\varphi_{a}-\delta\right)^{2}\left\|\nabla \log \left(1+\frac{\left\|s_{i}\right\|^{2}}{\varepsilon_{i}^{2}}\right)\right\|^{2} \omega^{n} \\
\leq & \frac{C}{a^{2}} \int_{\left\{\varphi_{a}>\delta\right\}}\left(\log \left(1+\frac{\left\|s_{i}\right\|^{2}}{\varepsilon_{i}^{2}}\right)\right)^{2} \omega^{n}+C q_{i} K_{0} \int_{\left\{\varphi_{a}>\delta\right\}} \log \left(1+\frac{\left\|s_{i}\right\|^{2}}{\varepsilon_{i}^{2}}\right) \omega^{n} \\
\leq & \frac{C}{a^{2} \varepsilon_{i}^{4}}\left(\sup _{M}\left\|s_{i}\right\|^{2}\right) \int_{\left\{\varphi_{a}>\delta\right\}}\left\|s_{i}\right\|^{2} \omega^{n}+\frac{C q_{i} K_{0}}{\varepsilon_{i}^{2}} \int_{\left\{\varphi_{a}>\delta\right\}}\left\|s_{i}\right\|^{2} \omega^{n} .
\end{aligned}
$$

Thus letting $\delta \rightarrow 0$ and $a \rightarrow+\infty$ we deduce the integral estimate

$$
\int_{M}\left\|\nabla \log \left(1+\frac{\left\|s_{i}\right\|^{2}}{\varepsilon_{i}^{2}}\right)\right\|^{2} \omega^{n} \leq \frac{C q_{i} K_{0}}{\varepsilon_{i}^{2}} \int_{M}\left\|s_{i}\right\|^{2} \omega^{n} .
$$

Then the first term on the RHS of (3.2) can be estimated as follows,

$$
\begin{aligned}
& \int_{\left\{\varphi_{a}>\delta\right\}} \log \left(1+\frac{\left\|s_{2}\right\|^{2}}{\varepsilon_{2}^{2}}\right) \sqrt{-1} \partial \bar{\partial}\left(\left(\varphi_{a}-\delta\right)^{3}\right) \wedge \zeta_{\varepsilon_{1}}\left(s_{1}\right) \wedge R i c^{n-2} \\
& \leq \frac{C}{a^{2}} \int_{\left\{\varphi_{a}>\delta\right\}}\left(\varphi_{a}-\delta\right) \log \left(1+\frac{\left\|s_{2}\right\|^{2}}{\varepsilon_{2}^{2}}\right) \cdot \sqrt{-1} \partial \bar{\partial} \log \left(\left\|s_{1}\right\|^{2}+\varepsilon_{1}^{2}\right) \\
& \wedge \omega \wedge R i c^{n-2}+\frac{C q_{1}}{a^{2}} \int_{\left\{\varphi_{a}>\delta\right\}}\left(\varphi_{a}-\delta\right) \log \left(1+\frac{\left\|s_{2}\right\|^{2}}{\varepsilon_{2}^{2}}\right) \omega \wedge R i c^{n-1} \\
& \leq \frac{C K_{0}^{n-2}}{a^{3}} \int_{\left\{\varphi_{a}>\delta\right\}} \log \left(1+\frac{\left\|s_{2}\right\|^{2}}{\varepsilon_{2}^{2}}\right) \cdot\left\|\nabla \log \left(\left\|s_{1}\right\|^{2}+\varepsilon_{1}^{2}\right)\right\| \omega^{n}+\frac{C K_{0}^{n-2}}{a^{2}} \\
& \quad \int_{\left\{\varphi_{a}>\delta\right\}}\left(\varphi_{a}-\delta\right)\left\|\nabla \log \left(\left\|s_{1}\right\|^{2}+\varepsilon_{1}^{2}\right)\right\| \cdot\left\|\nabla \log \left(\left\|s_{2}\right\|^{2}+\varepsilon_{2}^{2}\right)\right\| \omega^{n} \\
&+\frac{C q_{1} K_{0}^{n-1}}{a^{2}} \int_{\left\{\varphi_{a}>\delta\right\}}\left(\varphi_{a}-\delta\right) \log \left(1+\frac{\left\|s_{2}\right\|^{2}}{\varepsilon_{2}^{2}}\right) \omega^{n} \\
& \leq \frac{C K_{0}^{n-2}}{a^{3}} \int_{\left\{\varphi_{a}>\delta\right\}}\left(\log \left(1+\frac{\left\|s_{2}\right\|^{2}}{\varepsilon_{2}^{2}}\right)\right)^{2} \omega^{n}+\left(\frac{C K_{0}^{n-2}}{a^{3}}+\frac{C K_{0}^{n-2}}{a^{2}}\right) . \\
& \int_{\left\{\varphi_{a}>\delta\right\}}\left(\left\|\nabla \log \left(\left\|s_{1}\right\|^{2}+\varepsilon_{1}^{2}\right)\right\|^{2}+\left\|\nabla \log \left(\left\|s_{2}\right\|^{2}+\varepsilon_{2}^{2}\right)\right\|^{2}\right) \omega^{n} \\
&+\frac{C q_{1} K_{0}^{n-1}}{a^{2}} \int_{\left\{\varphi_{a}>\delta\right\}} \log \left(1+\frac{\left\|s_{2}\right\|^{2}}{\varepsilon_{2}^{2}}\right) \omega^{n}
\end{aligned}
$$




$$
\begin{gathered}
\leq \frac{C K_{0}^{n-2}}{a^{3} \varepsilon_{2}^{2}} \sup _{M}\left(\log \left(1+\frac{\left\|s_{2}\right\|^{2}}{\varepsilon_{2}^{2}}\right)\right) \int_{\left\{\varphi_{a}>\delta\right\}}\left\|s_{2}\right\|^{2} \omega^{n}+\frac{C K_{0}^{n-2}}{a^{2}}\left(1+\frac{1}{a}\right) \\
\quad \int_{\left\{\varphi_{a}>\delta\right\}}\left(\left\|\nabla \log \left(1+\frac{\left\|s_{1}\right\|^{2}}{\varepsilon_{1}^{2}}\right)\right\|^{2}+\left\|\nabla \log \left(1+\frac{\left\|s_{2}\right\|^{2}}{\varepsilon_{2}^{2}}\right)\right\|^{2}\right) \omega^{n} \\
+\frac{C q_{1} K_{0}^{n-1}}{a^{2} \varepsilon_{2}^{2}} \int_{\left\{\varphi_{a}>\delta\right\}}\left\|s_{2}\right\|^{2} \omega^{n} .
\end{gathered}
$$

Letting $\delta \rightarrow 0$ and $a \rightarrow+\infty$, we obtain from (3.2), (3.3), and (3.4) that

$$
\int_{M} \zeta_{\varepsilon_{1}}\left(s_{1}\right) \wedge \zeta_{\varepsilon_{2}}\left(s_{2}\right) \wedge R i c^{n-2} \leq C q_{1} q_{2} \int_{M} \operatorname{Ric}^{n}
$$

For the general case $k>2$, by inducting on $k$ and integrating by parts, we have

$$
\begin{aligned}
& \int_{\left\{\varphi_{a}>\delta\right\}}\left(\varphi_{a}-\delta\right)^{k+1} \zeta_{\varepsilon_{1}}\left(s_{1}\right) \wedge \cdots \wedge \zeta_{\varepsilon_{k}}\left(s_{k}\right) \wedge R i c^{n-k} \\
& =\int_{\left\{\varphi_{a}>\delta\right\}}\left(\varphi_{a}-\delta\right)^{k+1} \sqrt{-1} \partial \bar{\partial} \log \left(1+\frac{\left\|s_{k}\right\|^{2}}{\varepsilon_{k}^{2}}\right) \wedge \zeta_{\varepsilon_{1}}\left(s_{1}\right) \wedge \cdots \wedge \zeta_{\varepsilon_{k-1}}\left(s_{k-1}\right) \\
& \wedge \operatorname{Ric}^{n-k}+q_{k} \int_{\left\{\varphi_{a}>\delta\right\}}\left(\varphi_{a}-\delta\right)^{k+1} \zeta_{\varepsilon_{1}}\left(s_{1}\right) \wedge \cdots \wedge \zeta_{\varepsilon_{k-1}}\left(s_{k-1}\right) \wedge R i c^{n-k+1} \\
& \leq \frac{C}{a^{2}} \int_{\left\{\varphi_{a}>\delta\right\}}\left(\varphi_{a}-\delta\right)^{k-1} \log \left(1+\frac{\left\|s_{k}\right\|^{2}}{\varepsilon_{k}^{2}}\right) \zeta_{\varepsilon_{1}}\left(s_{1}\right) \wedge \cdots \wedge \zeta_{\varepsilon_{k-1}}\left(s_{k-1}\right) \wedge \\
& R i c^{n-k}+q_{k} \int_{\left\{\varphi_{a}>\delta\right\}}\left(\varphi_{a}-\delta\right)^{k+1} \zeta_{\varepsilon_{1}}\left(s_{1}\right) \wedge \cdots \wedge \zeta_{\varepsilon_{k-1}}\left(s_{k-1}\right) \wedge R i c^{n-k+1} \\
& \leq \frac{C}{a^{2}} \int_{\left\{\varphi_{a}>\delta\right\}}\left(\varphi_{a}-\delta\right)^{k-1} \log \left(1+\frac{\left\|s_{k}\right\|^{2}}{\varepsilon_{k}^{2}}\right) \sqrt{-1} \partial \bar{\partial} \log \left(1+\frac{\left\|s_{k-1}\right\|^{2}}{\varepsilon_{k-1}^{2}}\right) \wedge \\
& \zeta_{\varepsilon_{1}}\left(s_{1}\right) \wedge \cdots \wedge \zeta_{\varepsilon_{k-2}}\left(s_{k-2}\right) \wedge \omega \wedge R i c^{n-k} \\
& +\frac{C q_{k-1}}{a^{2}} \int_{\left\{\varphi_{a}>\delta\right\}}\left(\varphi_{a}-\delta\right)^{k-1} \log \left(1+\frac{\left\|s_{k}\right\|^{2}}{\varepsilon_{k}^{2}}\right) \zeta_{\varepsilon_{1}}\left(s_{1}\right) \wedge \cdots \wedge \\
& \zeta_{\varepsilon_{k-2}}\left(s_{k-2}\right) \wedge \omega \wedge R i c^{n-k+1}+C q_{1} \cdots q_{k} \int_{M} R i c^{n} \\
& \leq \frac{\widetilde{C}}{a^{2}} \int_{\left\{\varphi_{a}>\delta\right\}}\left(\varphi_{a}-\delta\right)^{k-2} \\
& \left(\left\|\nabla \log \left(1+\frac{\left\|s_{k-1}\right\|^{2}}{\varepsilon_{k-1}^{2}}\right)\right\|^{2}+\left\|\nabla \log \left(1+\frac{\left\|s_{k}\right\|^{2}}{\varepsilon_{k}^{2}}\right)\right\|^{2}+\log \left(1+\frac{\left\|s_{k}\right\|^{2}}{\varepsilon_{k}^{2}}\right)\right)
\end{aligned}
$$




$$
\wedge \zeta_{\varepsilon_{1}}\left(s_{1}\right) \wedge \cdots \wedge \zeta_{\varepsilon_{k-2}}\left(s_{k-2}\right) \wedge \omega^{n-k+2}+C q_{1} \cdots q_{k} \int_{M} R i c^{n} .
$$

Here and below we denote by $\widetilde{C}$ various positive constants depending only on $n, \varepsilon_{1}, \cdots, \varepsilon_{k}, q_{1}, \cdots, q_{k}, \sup _{M}\left\|s_{1}\right\|^{2}, \cdots, \sup _{M}\left\|s_{k}\right\|^{2}$ and $K_{0}$.

We now show that the following two estimates

$$
\int_{M} \log \left(1+\frac{\left\|s_{k}\right\|^{2}}{\varepsilon_{k}^{2}}\right) \zeta_{\varepsilon_{1}}\left(s_{1}\right) \wedge \cdots \wedge \zeta_{\varepsilon_{k-2}}\left(s_{k-2}\right) \wedge \omega^{n-k+2}<+\infty
$$

and

$$
\int_{M}\left\|\nabla \log \left(1+\frac{\left\|s_{k-1}\right\|^{2}}{\varepsilon_{k-1}^{2}}\right)\right\|^{2} \zeta_{\varepsilon_{1}}\left(s_{1}\right) \wedge \cdots \wedge \zeta_{\varepsilon_{k-2}}\left(s_{k-2}\right) \wedge \omega^{n-k+2}<+\infty
$$

hold by induction. The estimate (3.3) tells us that these integration are finite for the case $k=2$. Since

$$
\begin{aligned}
& \int_{\left\{\varphi_{a}>\delta\right\}}\left(\varphi_{a}-\delta\right)^{2} \log \left(1+\frac{\left\|s_{k}\right\|^{2}}{\varepsilon_{k}^{2}}\right) \zeta_{\varepsilon_{1}}\left(s_{1}\right) \wedge \cdots \wedge \zeta_{\varepsilon_{k-2}}\left(s_{k-2}\right) \wedge \omega^{n-k+2} \\
\leq & \frac{C}{a} \int_{\left\{\varphi_{a}>\delta\right\}}\left(\varphi_{a}-\delta\right) \log \left(1+\frac{\left\|s_{k}\right\|^{2}}{\varepsilon_{k}^{2}}\right)\left\|\nabla \log \left(1+\frac{\left\|s_{k-2}\right\|^{2}}{\varepsilon_{k-2}^{2}}\right)\right\| \\
& \zeta_{\varepsilon_{1}}\left(s_{1}\right) \wedge \cdots \wedge \zeta_{\varepsilon_{k-3}}\left(s_{k-3}\right) \wedge \omega^{n-k+3} \\
+ & \int_{\left\{\varphi_{a}>\delta\right\}}\left(\varphi_{a}-\delta\right)^{2}\left\|\nabla \log \left(1+\frac{\left\|s_{k}\right\|^{2}}{\varepsilon_{k}^{2}}\right)\right\|\left\|\nabla \log \left(1+\frac{\left\|s_{k-2}\right\|^{2}}{\varepsilon_{k-2}^{2}}\right)\right\| \\
& \zeta_{\varepsilon_{1}}\left(s_{1}\right) \wedge \cdots \wedge \zeta_{\varepsilon_{k-3}}\left(s_{k-3}\right) \wedge \omega^{n-k+3} \\
+ & q_{k-2} K_{0} \int_{\left\{\varphi_{a}>\delta\right\}}\left(\varphi_{a}-\delta\right)^{2} \log \left(1+\frac{\left\|s_{k}\right\|^{2}}{\varepsilon_{k}^{2}}\right) \zeta_{\varepsilon_{1}}\left(s_{1}\right) \wedge \cdots \wedge \\
& \zeta_{\varepsilon_{k-3}}\left(s_{k-3}\right) \wedge \omega^{n-k+3},
\end{aligned}
$$

it follows (3.6) directly by induction on the both estimates. To get (3.7), we recall that

$$
\zeta_{\varepsilon_{k-1}}\left(s_{k-1}\right)=\sqrt{-1} \partial \bar{\partial} \log \left(\left\|s_{k-1}\right\|^{2}+\varepsilon_{k-1}^{2}\right)+q_{k-1} R i c \geq 0 .
$$

Thus

$$
\begin{aligned}
\int_{\left\{\varphi_{a}>\delta\right\}}\left(\varphi_{a}-\delta\right)^{2} & \log \left(1+\frac{\left\|s_{k-1}\right\|^{2}}{\varepsilon_{k-1}^{2}}\right)\left(\sqrt{-1} \partial \bar{\partial} \log \left(\left\|s_{k-1}\right\|^{2}+\varepsilon_{k-1}^{2}\right)\right. \\
\left.+q_{k-1} \operatorname{Ric}\right) & \wedge \zeta_{\varepsilon_{1}}\left(s_{1}\right) \wedge \cdots \wedge \zeta_{\varepsilon_{k-2}}\left(s_{k-2}\right) \wedge \omega^{n-k+1} \geq 0 .
\end{aligned}
$$


Integrating by parts we have

$$
\begin{gathered}
\int_{\left\{\varphi_{a}>\delta\right\}}\left(\varphi_{a}-\delta\right)^{2} \sqrt{-1} \partial \log \left(1+\frac{\left\|s_{k-1}\right\|^{2}}{\varepsilon_{k-1}^{2}}\right) \wedge \bar{\partial} \log \left(1+\frac{\left\|s_{k-1}\right\|^{2}}{\varepsilon_{k-1}^{2}}\right) \wedge \\
\zeta_{\varepsilon_{1}}\left(s_{1}\right) \wedge \cdots \wedge \zeta_{\varepsilon_{k-2}}\left(s_{k-2}\right) \wedge \omega^{n-k+1} \\
\leq \frac{C}{a} \int_{\left\{\varphi_{a}>\delta\right\}}\left(\varphi_{a}-\delta\right) \log \left(1+\frac{\left\|s_{k-1}\right\|^{2}}{\varepsilon_{k-1}^{2}}\right)\left\|\nabla \log \left(1+\frac{\left\|s_{k-1}\right\|^{2}}{\varepsilon_{k-1}^{2}}\right)\right\| \zeta_{\varepsilon_{1}}\left(s_{1}\right) \wedge \\
\quad \cdots \wedge \zeta_{\varepsilon_{k-2}}\left(s_{k-2}\right) \wedge \omega^{n-k+2}+q_{k-1} K_{0} \cdot \\
\int_{\left\{\varphi_{a}>\delta\right\}}\left(\varphi_{a}-\delta\right)^{2} \log \left(1+\frac{\left\|s_{k-1}\right\|^{2}}{\varepsilon_{k-1}^{2}}\right) \zeta_{\varepsilon_{1}}\left(s_{1}\right) \wedge \cdots \wedge \zeta_{\varepsilon_{k-2}}\left(s_{k-2}\right) \wedge \omega^{n-k+2} .
\end{gathered}
$$

Applying Cauchy-Schwarz inequality, we get

$$
\begin{aligned}
& \int_{\left\{\varphi_{a}>\delta\right\}}\left(\varphi_{a}-\delta\right)^{2}\left\|\nabla \log \left(1+\frac{\left\|s_{k-1}\right\|^{2}}{\varepsilon_{k-1}^{2}}\right)\right\|^{2} \zeta_{\varepsilon_{1}}\left(s_{1}\right) \wedge \cdots \wedge \zeta_{\varepsilon_{k-2}}\left(s_{k-2}\right) \wedge \omega^{n-k+2} \\
& \leq \frac{C}{a^{2}} \int_{\left\{\varphi_{a}>\delta\right\}}\left(\log \left(1+\frac{\left\|s_{k-1}\right\|^{2}}{\varepsilon_{k-1}^{2}}\right)\right)^{2} \zeta_{\varepsilon_{1}}\left(s_{1}\right) \wedge \cdots \wedge \zeta_{\varepsilon_{k-2}}\left(s_{k-2}\right) \wedge \omega^{n-k+2} \\
& \quad+q_{k-1} K_{0} \int_{\left\{\varphi_{a}>\delta\right\}}\left(\varphi_{a}-\delta\right)^{2} \log \left(1+\frac{\left\|s_{k-1}\right\|^{2}}{\varepsilon_{k-1}^{2}}\right) \zeta_{\varepsilon_{1}}\left(s_{1}\right) \wedge \cdots \wedge \zeta_{\varepsilon_{k-2}}\left(s_{k-2}\right) \wedge \\
& \quad \omega^{n-k+2} \\
& \leq\left(\frac{\widetilde{C}}{a^{2}}+\widetilde{C}\right) \int_{M} \log \left(1+\frac{\left\|s_{k-1}\right\|^{2}}{\varepsilon_{k-1}^{2}}\right) \zeta_{\varepsilon_{1}}\left(s_{1}\right) \wedge \cdots \wedge \zeta_{\varepsilon_{k-2}}\left(s_{k-2}\right) \wedge \omega^{n-k+2}
\end{aligned}
$$

Hence we obtain (3.7) by using (3.6).

We thus let $\delta \rightarrow 0$ and $a \rightarrow+\infty$ in (3.5) and use (3.6) and (3.7) to obtain the desired estimate (3.1).

We will need to bound the Gauss-Bonnet integrals of curves which are the intersection of zero divisors of sections in $\bigcup_{q>0} \Gamma^{2}\left(M, K^{-q}\right)$. To this end we want to establish the associated Bézout estimate over projectivized tangent bundle.

We denote by $\pi: \mathbf{P} T M \rightarrow M$ the projectivied tangent bundle and $L$ the tautological line bundle. Fix a point $x_{0} \in M$, let $\left\{z_{1}, \cdots, z_{n}\right\}$ be a holomorphic 
coordinate system at $x_{0}$ with $z_{1}\left(x_{0}\right)=\cdots=z_{n}\left(x_{0}\right)=0$. For any tangent vector $v=v_{1} \frac{\partial}{\partial z_{1}}+\cdots+v_{n} \frac{\partial}{\partial z_{n}}$ with $v_{n} \neq 0$, let $u_{1}=\frac{v_{1}}{v_{n}}, \cdots, u_{n-1}=\frac{v_{n-1}}{v_{n}}$. Then $\left\{z_{1}, \cdots, z_{n}, u_{1}, \cdots, u_{n-1}\right\}$ forms a local holomorphic coordinate system on $\mathbf{P} T M$ at $\left(x_{0},\left[\frac{\partial}{\partial z_{1}}, \cdots, \frac{\partial}{\partial z_{n}}\right]\right)$. Equip $\mathbf{P} T M$ and $L$ with the induced metric from $M$. Then a direct computation ( see 15 ) gives

$$
c_{1}(L)=-\sqrt{-1} \sum_{1 \leq \gamma \leq n-1} d u^{\gamma} \wedge d \bar{u}^{\gamma}+\sqrt{-1} \sum_{1 \leq i, j \leq n} R_{n \bar{n} i \bar{j}} d z^{i} \wedge d \bar{z}^{j}
$$

where $c_{1}(L)$ is the first Chern form of the tautological line bundle. Since the sectional curvature of $M$ is positive, we thus have

$$
-c_{1}(L)+2 \cdot\left(\pi^{*} R i c\right) \geq \sum_{1 \leq \gamma \leq n-1} d u^{\gamma} \wedge d \bar{u}^{\gamma}+\sum_{1 \leq i, j \leq n} R_{i \bar{j}} d z^{i} \wedge d \bar{z}^{j}>0 .
$$

Hence $L^{*} \otimes \pi^{*} K^{-q}$ is a positive line bundle over $\mathbf{P} T M$ with bounded curvature for $p \geq 2$.

Set

$$
\nu=-c_{1}(L)+2 \cdot\left(\pi^{*} R i c\right)
$$

Now $\nu$ is a positive, closed (1.1) form on $\mathbf{P} T M$. Thus, $\nu$ can be regarded as a ( possible incomplete) Kähler metric on PTM.

Recall that $\varphi_{a}$ is the cut-off function in Lemma 3.1. Now we choose $\pi^{*} \varphi_{a}$ as a cut-off function on PTM. We remark that in the proof of Lemma 3.2 the main ingredients are the boundedness of the $L^{2}$ norm and $L^{\infty}$ norm of the section s. So one can proceed exactly as in the proof of Proposition 3.2 to obtain the following Bézout estimate.

Proposition 3.3 Let $(M, \omega)$ be assumed as in the Main Theorem. Let $s_{i} \in \Gamma^{2}\left(\mathbf{P} T M, \pi^{*} K^{-p_{i}}\right), i=1, \cdots, k, p_{1}, \cdots, p_{k} \geq 2$ and $t_{j} \in \Gamma^{2}\left(\mathbf{P} T M, L^{*} \otimes\right.$ $\left.\pi^{*} K^{-q_{j}}\right), j=1, \cdots, l, q_{1}, \cdots, q_{l} \geq 2$. Suppose also that all $\left\|s_{i}\right\|$ and $\left\|t_{j}\right\|$ are bounded. For any sequence $\varepsilon_{i}>0, i=1, \cdots, k+l$, define

$$
\zeta_{\varepsilon_{i}}\left(s_{i}\right)=\sqrt{-1} \partial \bar{\partial} \log \left(\left\|s_{i}\right\|^{2}+\varepsilon_{i}^{2}\right)+c_{1}\left(\pi^{*} K^{-p_{i}}\right), \quad i=1, \cdots, k
$$


and

$$
\eta_{\varepsilon_{j+k}}\left(t_{j}\right)=\sqrt{-1} \partial \bar{\partial} \log \left(\left\|t_{j}\right\|^{2}+\varepsilon_{j+k}^{2}\right)+c_{1}\left(L^{*} \otimes \pi^{*} K^{-q_{j}}\right), \quad j=1, \cdots, l,
$$

where $c_{1}\left(\pi^{*} K^{-p_{i}}\right)$ and $c_{1}\left(L^{*} \otimes \pi^{*} K^{-q_{i}}\right)$ are the first Chern forms of the corresponding line bundles. Then

$$
\begin{aligned}
& \int_{\mathbf{P} T M} \zeta_{\varepsilon_{1}}\left(s_{1}\right) \wedge \cdots \wedge \zeta_{\varepsilon_{k}}\left(s_{k}\right) \wedge \eta_{\varepsilon_{k+1}}\left(t_{1}\right) \wedge \cdots \wedge \eta_{\varepsilon_{k+l}}\left(t_{l}\right) \wedge v^{2 n-1-k-l} \\
\leq & C p_{1} \cdots p_{k} q_{1} \cdots q_{l} \int_{\mathbf{P} T M} \nu^{2 n-1}
\end{aligned}
$$

where $C$ is a positive constant depending only on $n$.

We remark that $\mathbf{P} T M$ has finite volume with respect to the Kähler metric $\nu$, i.e.,

$$
\begin{aligned}
\int_{\mathbf{P} T M} \nu^{2 n-1} & \leq \text { Const } \cdot \int_{M}\left(\int_{\mathbf{P} T_{x} M} \omega_{F S}\right) \operatorname{Ric}^{n} \\
& \leq \text { Const } \cdot \operatorname{Vol}\left(\mathbf{P}^{n-1}\right) \cdot \int_{M} \operatorname{Ric}^{n}<+\infty
\end{aligned}
$$

where we denote by $\omega_{F S}$ the Fubini-Study metric on $\mathbf{P}^{n-1}$.

We are ready to derive an estimate for the Gauss-Bonnet integrals of curves which are the intersection of zero divisors of section in $\bigcup_{q>0} \Gamma^{2}\left(M, K^{-q}\right)$.

For any smooth holomorphic curve $S$ on $M$, let $\widehat{\theta}: S \rightarrow \mathbf{P} T M$ denote the lifting of $S$ to $\mathbf{P} T M$ defined by $\widehat{\theta}(x)=\left[T_{x} S\right]$ for $x \in S$, where $\left[T_{x} S\right]$ denotes the element in PTM defined by $T_{x} S$. Let $\widehat{S}$ denote the image $\widehat{\theta}(S)$. Suppose $S$ lies in the intersection of zero divisors of $n-1$ holomorphic sections $t_{1}, \cdots, t_{n-1}$, where $t_{i} \in \Gamma^{2}\left(M, K^{-q}\right)$ ( for some positive integer $q \geq 2$ ). Without loss of generality, we may assume that $q$ is sufficiently large. By $L^{2}$-estimate of $\bar{\partial}$ operator as in Section 2, we can choose an $L^{2}$ holomorphic plurianticanonical section $t_{0}$ of the same degree $q$ such that $t_{0}$ does not vanish identically on each zero divisor of holomorphic section $t_{i}, 1 \leq i \leq n-1$. As shown in Section 4 of [15], all $d\left(t_{i} / t_{0}\right) \cdot t_{0}^{2}=t_{0} \nabla t_{i}-t_{i} \nabla t_{0}$ are holomorphic sections of $L^{*} \otimes \pi^{*} K^{-2 q}$ over $\mathbf{P} T M$ for $i=1, \cdots, n-1$, and $\widehat{S}$ lies in the intersection of $2 n-2$ zero divisors 
of $\pi^{*} t_{1}, \cdots, \pi^{*} t_{n-1}, t_{0} \nabla t_{1}-t_{1} \nabla t_{0}, \cdots, t_{0} \nabla t_{n-1}-t_{n-1} \nabla t_{0}$. In order to apply Proposition 3.3, we will show that all $\left\|\nabla t_{i}\right\|, i=0, \cdots, n-1$, are bounded on $M$. Now we use the Ricci flow to get the following gradient estimate for $L^{2}$ holomorphic plurianticanonical sections, which has its own interest in analysis.

Proposition 3.4 Suppose $M$ has positive and bounded sectional curvature. Let $s$ be a holomorphic section belonging $\Gamma^{2}\left(M, K^{-q}\right)$. Then $\|\nabla s\|$ is bounded on $M$.

Proof. Let $\left(z_{1}, \cdots, z_{n}\right)$ be a local holomorphic coordinate system and write

$$
s=f\left(\frac{\partial}{\partial z_{1}} \wedge \cdots \wedge \frac{\partial}{\partial z_{n}}\right)^{q}
$$

locally for some holomorphic function $f$. Then $h=\operatorname{det}\left(g_{\alpha \bar{\beta}}\right)^{q}$ is the Hermitian metric on $K^{-q}$ and $C(L)=-\sqrt{-1} \partial \bar{\partial} \log h=q R i c$ is the curvature form of $L=K^{-q}$ with respect to the coordinates $z_{1}, \cdots, z_{n}$. The covariant derivative of $s$ is given by

$$
\nabla s=\left(\frac{\partial f}{\partial z_{i}}+q f \frac{\partial}{\partial z_{i}} \log \operatorname{det}\left(g_{\alpha \bar{\beta}}\right)\right) \otimes d z_{i} \otimes\left(\frac{\partial}{\partial z_{i}} \wedge \cdots \wedge \frac{\partial}{\partial z_{n}}\right)^{q} .
$$

We use the Bochner trick to bound $\nabla s$. Let $\left(z_{1}, \cdots, z_{n}\right)$ be a normal holomorphic coordinate at a fixed point. We compute

$\|\nabla s\|^{2}=g^{i \bar{j}}\left(\frac{\partial f}{\partial z_{i}}+q f \frac{\partial}{\partial z_{i}} \log \operatorname{det}\left(g_{\alpha \bar{\beta}}\right)\right) \overline{\left(\frac{\partial f}{\partial z_{j}}+q f \frac{\partial}{\partial z_{j}} \log \operatorname{det}\left(g_{\alpha \bar{\beta}}\right)\right)} \operatorname{det}\left(g_{\alpha \bar{\beta}}\right)^{q}$, 


$$
\begin{aligned}
& \frac{\partial}{\partial \bar{z}_{l}}\|\nabla s\|^{2} \\
& =\frac{\partial g^{i \bar{j}}}{\partial \bar{z}_{l}}\left(\frac{\partial f}{\partial z_{i}}+q f \frac{\partial}{\partial z_{i}} \log \operatorname{det}\left(g_{\alpha \bar{\beta}}\right)\right) \overline{\left(\frac{\partial f}{\partial z_{j}}+q f \frac{\partial}{\partial z_{j}} \log \operatorname{det}\left(g_{\alpha \bar{\beta}}\right)\right)} \operatorname{det}\left(g_{\alpha \bar{\beta}}\right)^{q} \\
& +\frac{\partial \operatorname{det}\left(g_{\alpha \bar{\beta}}\right)^{q}}{\partial \bar{z}_{l}} g^{i \bar{j}}\left(\frac{\partial f}{\partial z_{i}}+q f \frac{\partial}{\partial z_{i}} \log \operatorname{det}\left(g_{\alpha \bar{\beta}}\right)\right) \overline{\left(\frac{\partial f}{\partial z_{j}}+q f \frac{\partial}{\partial z_{j}} \log \operatorname{det}\left(g_{\alpha \bar{\beta}}\right)\right)} \\
& +g^{i \bar{j}}\left(q f \frac{\partial^{2}}{\partial z_{i} \partial \bar{z}_{l}} \log \operatorname{det}\left(g_{\alpha \bar{\beta}}\right)\right) \overline{\left(\frac{\partial f}{\partial z_{j}}+q f \frac{\partial}{\partial z_{j}} \log \operatorname{det}\left(g_{\alpha \bar{\beta}}\right)\right)} \operatorname{det}\left(g_{\alpha \bar{\beta}}\right)^{q} \\
& +g^{i \bar{j}}\left(\frac{\partial f}{\partial z_{i}}+q f \frac{\partial}{\partial z_{i}} \log \operatorname{det}\left(g_{\alpha \bar{\beta}}\right)\right) \cdot \\
& \overline{\left(\frac{\partial^{2} f}{\partial z_{j} \partial z_{l}}+q f \frac{\partial^{2}}{\partial z_{j} \partial z_{l}} \log \operatorname{det}\left(g_{\alpha \bar{\beta}}\right)+q \frac{\partial f}{\partial z_{l}} \frac{\partial}{\partial z_{j}} \log \operatorname{det}\left(g_{\alpha \bar{\beta}}\right)\right)} \operatorname{det}\left(g_{\alpha \bar{\beta}}\right)^{q},
\end{aligned}
$$

and

$$
\begin{aligned}
& \frac{\partial^{2}}{\partial z_{k} \partial \bar{z}_{l}}\|\nabla s\|^{2} \\
& =R_{k \bar{l} \bar{j}}\left(\frac{\partial f}{\partial z_{i}}+q f \frac{\partial}{\partial z_{i}} \log \operatorname{det}\left(g_{\alpha \bar{\beta}}\right)\right) \overline{\left(\frac{\partial f}{\partial z_{j}}+q f \frac{\partial}{\partial z_{j}} \log \operatorname{det}\left(g_{\alpha \bar{\beta}}\right)\right)} \operatorname{det}\left(g_{\alpha \bar{\beta}}\right)^{q} \\
& +\frac{\partial^{2} \operatorname{det}\left(g_{\alpha \bar{\beta}}\right)^{q}}{\partial z_{k} \partial \bar{z}_{l}} \cdot g^{i \bar{j}}\left(\frac{\partial f}{\partial z_{i}}+q f \frac{\partial}{\partial z_{i}} \log \operatorname{det}\left(g_{\alpha \bar{\beta}}\right)\right) \overline{\left(\frac{\partial f}{\partial z_{j}}+q f \frac{\partial}{\partial z_{j}} \log \operatorname{det}\left(g_{\alpha \bar{\beta}}\right)\right)} \\
& +g^{i \bar{j}}\left(q \frac{\partial f}{\partial z_{k}} \frac{\partial^{2}}{\partial z_{i} \partial \bar{z}_{l}} \log \operatorname{det}\left(g_{\alpha \bar{\beta}}\right)+q f \frac{\partial^{3}}{\partial z_{i} \partial \bar{z}_{l} \partial z_{k}} \log \operatorname{det}\left(g_{\alpha \bar{\beta}}\right)\right) \times \\
& \overline{\left(\frac{\partial f}{\partial z_{j}}+q f \frac{\partial}{\partial z_{j}} \log \operatorname{det}\left(g_{\alpha \bar{\beta}}\right)\right)} \operatorname{det}\left(g_{\alpha \bar{\beta}}\right)^{q} \\
& +g^{i \bar{j}}\left(q f \frac{\partial^{2}}{\partial z_{i} \partial \bar{z}_{l}} \log \operatorname{det}\left(g_{\alpha \bar{\beta}}\right)\right) \overline{\left(q f \frac{\partial^{2}}{\partial \bar{z}_{k} \partial z_{j}} \log \operatorname{det}\left(g_{\alpha \bar{\beta}}\right)\right)} \operatorname{det}\left(g_{\alpha \bar{\beta}}\right)^{q} \\
& +g^{i \bar{j}}\left(\frac{\partial^{2} f}{\partial z_{i} \partial z_{k}}+q \frac{\partial f}{\partial z_{k}} \frac{\partial}{\partial z_{i}} \log \operatorname{det}\left(g_{\alpha \bar{\beta}}\right)+q f \frac{\partial^{2}}{\partial z_{i} \partial z_{k}} \log \operatorname{det}\left(g_{\alpha \bar{\beta}}\right)\right) \times
\end{aligned}
$$




$$
\begin{aligned}
& \overline{\left(\frac{\partial^{2} f}{\partial z_{j} \partial z_{l}}+q f \frac{\partial^{2}}{\partial z_{j} \partial z_{l}} \log \operatorname{det}\left(g_{\alpha \bar{\beta}}\right)+q \frac{\partial f}{\partial z_{l}} \frac{\partial}{\partial z_{j}} \log \operatorname{det}\left(g_{\alpha \bar{\beta}}\right)\right)} \operatorname{det}\left(g_{\alpha \bar{\beta}}\right)^{q} \\
+ & g^{i \bar{j}}\left(\frac{\partial f}{\partial z_{i}}+q f \frac{\partial}{\partial z_{i}} \log \operatorname{det}\left(g_{\alpha \bar{\beta}}\right)\right) \times \\
& \overline{\left(q \frac{\partial f}{\partial z_{l}} \frac{\partial^{2}}{\partial \bar{z}_{k} \partial z_{j}} \log \operatorname{det}\left(g_{\alpha \bar{\beta}}\right)+q f \frac{\partial^{3}}{\partial \bar{z}_{k} \partial z_{l} \partial z_{j}} \log \operatorname{det}\left(g_{\alpha \bar{\beta}}\right)\right)} \operatorname{det}\left(g_{\alpha \bar{\beta}}\right)^{q} .
\end{aligned}
$$

Thus we obtain

$$
\begin{aligned}
\triangle\|\nabla s\|^{2}= & \left\|\nabla^{2} s\right\|^{2}+\operatorname{Ric}(\nabla s, \nabla s)-\operatorname{trace} C(L)\|\nabla s\|^{2}+\|C(L)\|^{2} \cdot\|s\|^{2} \\
& +2 \operatorname{Re}\langle\nabla s, \nabla(\operatorname{trace} C(L)) \otimes s\rangle-2 C(L)(\nabla s, \nabla s) .
\end{aligned}
$$

Suppose the sectional curvature is bounded by a positive constant $K_{0}$. Thus the above Bochner formula simplifies to

$$
\begin{aligned}
\Delta\|\nabla s\|^{2} & \geq-C q K_{0}\|\nabla s\|^{2}-C q\|\nabla s\| \cdot\|s\| \cdot\|\nabla R\| \\
& \geq-C\|\nabla s\|^{2}-\|s\|^{2} \cdot\|\nabla R\|^{2} .
\end{aligned}
$$

From Proposition 2.3 we know that $\|s\|$ is bounded and $\|\nabla s\|$ is squareintegrable. But the term containing $\|\nabla R\|$ cause the difficulty to derive a pointwise estimate for $\|\nabla s\|$ by using the mean value inequality since at a priori we have no control on $\|\nabla R\|$. To overcome the difficulty we consider the Hamilton's Ricci flow equation on $M$,

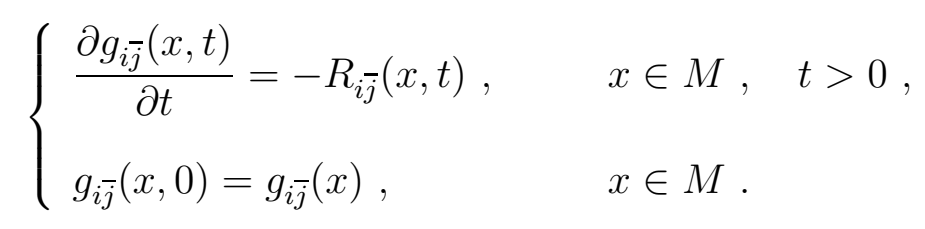

We may assume that the curvature tensor $R_{m}$ is also bounded by the positive constant $K_{0}$. From [21] we know that there exists a constant $\delta>0$, depending only on $K_{0}$ and the dimension $n$, such that the Ricci flow equation (3.14) has a solution $g_{i \bar{j}}(x, t)$ on $M \times[0, \delta]$ with the following estimates

$$
\frac{1}{2} g_{i \bar{j}}(\cdot) \leq g_{i \bar{j}}(\cdot, t) \leq 2 g_{i \bar{j}}(\cdot),
$$




$$
\begin{gathered}
\left\|R_{m}(\cdot, t)\right\|_{t} \leq 2 K_{0}, \\
\left\|\nabla^{t} R_{m}(\cdot, t)\right\|_{t} \leq \frac{C}{\sqrt{t}},
\end{gathered}
$$

where $\|\cdot\|_{t}$ and $\nabla^{t}$ are the norm and the covariant derivature with respect to the metric $g_{i \bar{j}}(\cdot, t)$, the constant $C$ depending only on $K_{0}$ and the dimension.

Denote by $B_{t}\left(x_{0}, 1\right)$ the geodesic ball of radius 1 and $\operatorname{Vol}_{t}\left(B_{t}\left(x_{0}, 1\right)\right)$ its volume with respect to the metric $g_{i \bar{j}}(\cdot, t)$. Since the sectional curvature is positive and bounded at $t=0$, this implies that the injectivity radius has a positive lower bound and then we have the following estimate

$$
\operatorname{Vol}\left(B_{0}\left(x_{0}, \frac{1}{\sqrt{2}}\right)\right) \geq \beta>0
$$

for some positive constant depending only on $K_{0}$ and $n$. By using (3.15), it is easy to see

$$
\begin{aligned}
\operatorname{Vol}_{t}\left(B_{t}\left(x_{0}, 1\right)\right) & \geq \int_{B_{0}\left(x_{0}, \frac{1}{\sqrt{2}}\right)} \operatorname{det}\left(g_{i j}(\cdot, t)\right) \\
& \geq \frac{1}{2^{n}} \operatorname{Vol}\left(B_{0}\left(x_{0}, \frac{1}{\sqrt{2}}\right)\right) \\
& \geq \frac{\beta}{2^{n}} .
\end{aligned}
$$

Furthermore, we know from [21] that the positivity of holomorphic bisectional curvature is preserved under the Ricci flow (3.14). In particular, the Ricci curvature is positive for $t \in[0, \delta]$.

Note that $\|s\|$ is bounded and square-integrable. The estimate (3.15) simply says that the metrics $g_{i j}(\cdot, t)$ are equivalent for $t \in[0, \delta]$. It follows that $s$ is still bounded and square-integrable holomorphic section of $K^{-q}$ for $t \in[0, \delta]$. Then Proposition 2.3 gives us the $L^{2}$ gradient estimate

$$
\left\|\nabla^{t} s\right\|_{L^{2}\left(g_{i \bar{j}}(\cdot, t)\right)}<+\infty .
$$

On the other hand, by (3.13), (3.16), and (3.17), we have

$$
\triangle_{\delta}\left\|\nabla^{\delta} s\right\|_{\delta}^{2} \geq-B\left\|\nabla^{\delta} s\right\|_{\delta}^{2}-B
$$


for some positive constant $B$, where $\triangle_{s}$ is the Laplacian operator of the metric $g_{i \bar{j}}(\cdot, \delta)$. Consider $M$ equipped with the metric $g_{i \bar{j}}(\delta)$ and let $G=$ $e^{\sqrt{B} \tau}\left(\left\|\nabla^{\delta} s\right\|_{\delta}^{2}+1\right)$ be a function defined on the product manifold $\widetilde{M}=M \times \mathbf{R}$, equipped with the product metric. Let $\widetilde{\triangle}=\triangle_{\delta}+\frac{\partial^{2}}{\partial \tau^{2}}$ be the Laplacian operator. It is clear that $\widetilde{M}$ has nonnegative Ricci curvature and we have

$$
\widetilde{\triangle} G \geq 0
$$

From (3.18) and (3.19), by using the mean value inequality for the subharmonic function $G$ on $\widetilde{M}$ exactly as in the proof of Proposition 2.3, we see that

$$
\sup _{x \in M}\left\|\nabla^{\delta} s\right\|_{\delta}^{2}(x)<+\infty .
$$

By (3.12) we have

$$
\begin{aligned}
\nabla^{\delta} s-\nabla s & =q f \frac{\partial}{\partial z_{i}} \log \frac{\operatorname{det}\left(g_{\alpha \bar{\beta}}(\cdot, \delta)\right)}{\operatorname{det}\left(g_{\alpha \bar{\beta}}(\cdot, 0)\right)} d z_{i} \otimes\left(\frac{\partial}{\partial z_{1}} \wedge \cdots \wedge \frac{\partial}{\partial z_{n}}\right)^{q} \\
& =q \partial F \otimes s
\end{aligned}
$$

where $F=\log \operatorname{det}\left(g_{\alpha \bar{\beta}}(\cdot, \delta)\right)-\log \operatorname{det}\left(g_{\alpha \bar{\beta}}(\cdot, 0)\right)$. By using the Ricci flow equation (3.14) one readily sees that

$$
F(x, t)=-\int_{0}^{\delta} R(x, t) d t .
$$

Thus by combining (3.15) and (3.17), we have

$$
\begin{aligned}
\|\partial F\|_{0} & \leq \sqrt{2} \int_{0}^{\delta}\left\|\nabla^{t} R(\cdot, t)\right\|_{t} d t \\
& \leq \sqrt{2} \int_{0}^{\delta} \frac{C}{\sqrt{t}} d t \\
& \leq \text { const. }
\end{aligned}
$$

which implies that

$$
\left\|\nabla^{\delta} s-\nabla s\right\|_{0} \leq \text { const. }\|s\|_{0}
$$


and then

$$
\begin{aligned}
\|\nabla s\|_{0} & \leq\left\|\nabla^{\delta} s-\nabla s\right\|_{0}+\left\|\nabla^{\delta} s\right\|_{0} \\
& \leq \text { const. }\|s\|_{0}+2^{\frac{n q+1}{2}}\left\|\nabla^{\delta} s\right\|_{\delta} \\
& <+\infty
\end{aligned}
$$

by (3.15) and (3.20). Therefore we have completed the proof of the proposition.

We now can apply Proposition 3.3 to the smooth holomorphic curve $S$ which lies in the intersection of the zero divisors of the $n-1$ holomorphic sections $t_{1}, \cdots, t_{n-1}$, where $t_{i} \in \Gamma^{2}\left(M, K^{-q}\right)$.

By using Proposition 3.3 and (3.11) we see that

$$
\begin{aligned}
\int_{\mathbf{P} T M} \zeta_{\varepsilon_{1}}\left(\pi^{*} t_{1}\right) \wedge \cdots & \wedge \zeta_{\varepsilon_{n-1}}\left(\pi^{*} t_{n-1}\right) \wedge \eta_{\varepsilon_{n}}\left(t_{0} \nabla t_{1}-t_{1} \nabla t_{0}\right) \\
& \wedge \cdots \wedge \eta_{\varepsilon_{2 n-2}}\left(t_{0} \nabla t_{n-1}-t_{n-1} \nabla t_{0}\right) \wedge \nu \leq C q^{2 n-2} \int_{M} R i c^{n},
\end{aligned}
$$

and by Fatou's Lemma and the Poincaré-Lelong equation,

$$
\int_{\widehat{S}} \nu \leq C q^{2 n-2} \int_{M} \operatorname{Ric}^{n}<+\infty
$$

On the other hand, a calculation as in Mok [15] easily leads to the following formula

$$
\int_{\widehat{S}} \nu=-\int_{S} K(x)+2 \int_{S} R i c
$$

where $K(x)$ is the Gaussian curvature of $S$. Hence we have

$$
\int_{S} K(x) \geq-C q^{2 n-2} \int_{M} R i c^{n}
$$

where $C$ is a positive constant depending only on $n$. Thus we prove the following result.

Proposition 3.5 Let $M$ be assumed as in the Main Theorem. Suppose $S$ is a smooth holomorphic curve which lies in the intersection of $n-1$ zero divisors of $t_{1}, \cdots, t_{n-1} \in \Gamma^{2}\left(M, K^{-q}\right),(q \geq 2)$. Then the Gauss-Bonnet integral 
satisfies,

$$
\int_{S} K(x) \geq-C q^{2 n-2} \int_{M} R i c^{n},
$$

where $C$ is a positive constant depending only on $n$.

\section{§4. Proof of the Main Theorem}

This section is devoted to the proof of the Main Theorem in the introduction. Let $M$ be assumed as in the Main Theorem. From Section 2 we know that the space $\bigcup_{q>0} \Gamma^{2}\left(M, K^{-q}\right)$ forms a $\mathbf{Z}^{+}$-graded algebra, gives local holomorphic coordinates at any point in $M$ and separates points of $M$. Applying Fatou's lemma to Proposition 3.2 with $k=1$, we have

$$
\int_{[s=0]} \operatorname{Ric}^{n-1} \leq \liminf _{\varepsilon \rightarrow 0} \int_{M} \zeta_{\varepsilon}(s) \wedge R i c^{n-1} \leq C q \int_{M} \operatorname{Ric}^{n}
$$

for any $s \in \Gamma^{2}\left(M, K^{-q}\right)$, where $C$ is a positive constant depending only on $n$, and $[s=0]$ denotes the zero divisor of $s$ counting multiplicity. Fix a point $x_{0} \in M$. Since the Ricci curvature is positive, it follows from (4.1) and the inequality of Bishop-Lelong that the multiplicity estimate

$$
\text { mult }\left([s=0], x_{0}\right) \leq C_{1} q
$$

holds for all $s \in \Gamma^{2}\left(M, K^{-q}\right)$, where the positive constant $C_{1}$ may depend on $x_{0}$ but independent of $s$ and $q$. Then it follows from a standard argument by considering the local Taylor expansion of $s \in \Gamma^{2}\left(M, K^{-q}\right)$ at the fixed point $x_{0}$ ( c.f. the proof of Proposition 5.1 in [14] ) that

$$
\operatorname{dim}_{\mathbf{C}} \Gamma^{2}\left(M, K^{-q}\right) \leq C_{2} q^{n}
$$

where $C_{2}$ is independent of $q$.

Denote by $R\left(M, K^{-1}\right)$ the subset of meromorphic functions on $M$ obtained by taking quotients in $\bigcup_{q>0} \Gamma^{2}\left(M, K^{-q}\right)$ of the same degree, i.e.,

$$
R\left(M, K^{-1}\right)=\left\{\frac{s}{t} \mid s, t \in \Gamma^{2}\left(M, K^{-q}\right), \text { where } q \in \mathbf{Z}^{+} \text {and } t \not \equiv 0\right\} .
$$


Since $\bigcup_{q>0} \Gamma^{2}\left(M, K^{-q}\right)$ forms a graded algebra under addition and multiplication, it thus is easy to see that $R\left(M, K^{-1}\right)$ forms a subfield of the field of meromorphic functions on $M$. Let $s_{0}, s_{1}, \cdots, s_{n}$ be $n+1$ holomorphic sections in $\Gamma^{2}\left(M, K^{-q}\right)$ ( for some $q>0$ large enough ) constructed in Section 2 such that $s_{1} / s_{0}, \cdots, s_{n} / s_{0}$ give a local holomorphic coordinate system at $x_{0}$. By the estimate (4.2), the classical argument of Poincaré-Siegel ( c.f. e.g. Mok [14 ) shows that $R\left(M, K^{-1}\right)$ is a finite extension field over $\mathbf{C}\left(\frac{s_{1}}{s_{0}}, \cdots, \frac{s_{n}}{s_{0}}\right)$. And by the primitive element theorem, the subfield $R\left(M, K^{-1}\right)$ is given by

$$
R\left(M, K^{-1}\right)=\mathbf{C}\left(\frac{s_{1}}{s_{0}}, \cdots, \frac{s_{n}}{s_{0}}, g\right),
$$

where $g$ is some meromorphic function in $R\left(M, K^{-1}\right)$ and is algebraic over $\mathbf{C}\left(\frac{s_{1}}{s_{0}}, \cdots, \frac{s_{n}}{s_{0}}\right)$. Also by taking common denominators we may assume $g=$ $s_{n+1} / s_{0}$, where $s_{0}, s_{1}, \cdots, s_{n}, s_{n+1} \in \Gamma^{2}\left(M, K^{-q}\right)$ for some $q>0$ large enough. Now consider the mapping $F: M \rightarrow \mathbf{P}^{n+1}$ defined by

$$
F(x)=\left[s_{0}(x), s_{1}(x), \cdots, s_{n}(x)\right], \quad \text { for } \quad x \in M
$$

Since $g$ is algebraic, the minimal equation satisfied by $g$ over $\mathbf{C}\left(\frac{s_{1}}{s_{0}}, \cdots, \frac{s_{n}}{s_{0}}\right)$ can be given by

$$
g^{p}+\sum_{0 \leq j \leq p-1} R_{j}\left(\frac{s_{1}}{s_{0}}, \cdots, \frac{s_{n}}{s_{0}}\right) g^{j}=0,
$$

where $R_{j}, 1 \leq j \leq p-1$, are rational functions of $n$ variables. After clearing denominators, we see that $s_{0}, s_{1}, \cdots, s_{n+1}$ satisfy a homogeneous equation

$$
P\left(s_{0}, s_{1}, \cdots, s_{n+1}\right)=0
$$

Let $Z_{0}$ be the hypersurface of $\mathbf{P}^{n+1}$ defined by

$$
Z_{0}=\left\{\left[s_{0}, s_{1}, \cdots, s_{n+1}\right] \in \mathbf{P}^{n+1} \mid P\left(s_{0}, s_{1}, \cdots, s_{n+1}\right)=0\right\}
$$

and let $Z$ be the connected component of $Z_{0}$ containing $F(M \backslash Q)$ where $Q$ is the set of indeterminancy of $F$. 
In the following we will show that $F$ is an "almost injective" and "almost surjective" map to $Z$ and we can desingularize $F$ to obtain a biholomorphic map from $M$ onto a quasi-projective variety by adjoining a finite number of holomorphic plurianticanonical sections.

First of all, we claim that $Z$ is irreducible and $F$ is "almost injective", i.e., there exists a subvariety $V$ of $M$ such that $\left.F\right|_{M \backslash V}: M \backslash V \rightarrow Z$ is an injective locally biholomorphic mapping. Indeed, take $V$ to be the union of the branching locus and the base locus of $F$ and $F^{-1}(\operatorname{Sing}(Z))$, here $\operatorname{Sing}(Z)$ denotes the singular set of $Z$. It is clear that $F$ is locally biholomorphism on $M \backslash V$. That $F$ is also injective there follows from the fact that $\bigcup_{q>0} \Gamma^{2}\left(M, K^{-q}\right)$ separates points and $s_{1} / s_{0}, \cdots, s_{n} / s_{0}, s_{n+1} / s_{0}$ generate $R\left(M, K^{-1}\right)$. To see the irreducibility of $Z$, note that $M \backslash(Q \cup(\operatorname{Sing} Z))$ is connected since $Q \cup(\operatorname{Sing} Z)$ has real codimension 2. Here $\overline{F(M \backslash(Q \cup(\operatorname{Sing} Z)))}$ is irreducible ( as its set of smooth points is connected ). Since $F(M \backslash Q) \subset \overline{F(M \backslash(Q \cup(\operatorname{Sing} Z)))}$, by the definition of $Z$, it must be irreducible.

We now make a remark for the subvariety $Z$. We have seen that $F$ is a biholomorphic embedding from $M \backslash V$ into its image $F(M \backslash V)$ in $Z$. As in Mok [15], $F$ is a birational mapping from $M$ to $Z$ with respect to the subfield $R\left(M, K^{-1}\right)$, i.e., the rational function field over $Z$ is isomorphic to $R\left(M, K^{-1}\right)$ via the pull back $F^{*}$. Therefore for any projective desingularization $\pi: Z^{\prime} \rightarrow$ $Z$, where $Z^{\prime}$ is a smooth projective variety, $\pi^{-1} \circ F$ is still a birational mapping from $M$ to $Z^{\prime}$ with respect to $R\left(M, K^{-1}\right)$. Since $\pi^{-1}$ is a birational map ( in the usual sense ), the composition $\pi^{-1} \circ F$ is easily seen to be defined by sections in $\Gamma^{2}\left(M, K^{-q}\right)$ for some large positive integer. Henceforth at each step when we desingularize $F$ by adding plurianticanonical sections, we may assume $Z$ is smooth.

Next, we come to the "almost surjectivity" of $F$, i.e., the image $F(M \backslash V)$ contains a Zariski-open subset of $Z$. Since the sectional curvature of $M$ is 
positive, by the result of Greene and $\mathrm{Wu}$ [8, $M$ is Stein. Thus as in MokZhong [17 ( see also To [26] ) by using Simha's criterion 22 for a domain of holomorphy contained in the unit polydisc to be Zariski open, the proof of the "almost surjectivity" of $F$ is reduced to show that the cardinality of the intersections of the complement of $F(M \backslash V)$ with the generic curves obtained by intersecting $Z$ with $(n-1)$ hyperplane sections of $\mathbf{P}^{n+1}$ are bounded. The following argument is basically due to Mok-Zhong ( see Proposition (2.2.2) and Proposition (2.2.3) in [17]).

Let $S_{0} \subset Z$ be a curve obtained by intersecting $Z$ with $(n-1)$ hyperplane sections, which correspond to $(n-1)$ holomorphic sections, say $t_{1}, \cdots, t_{n-1}$, in $\Gamma^{2}\left(M, K^{-q}\right)$. Assume that the hyperplane sections and $Z$ intersect at normal crossings at $Q \in Z$ and denote by $S$ the irreducible component of $S_{0}$ containing $Q$. Let $D$ denote $F(M \backslash V) \cap S$ and assume that $D$ is nonempty for the generic $S$. Define $\Sigma$ to be the closure of $F^{-1}(D)$ on $M$ and $f$ to be the meromorphic extension of $\left.F\right|_{F^{-1}(D)}$ to $\Sigma$. In order to prove that the cardinality of $S \backslash D$ is bounded, we proceed to prove that $f(\Sigma) \backslash D$ and $S \backslash f(\Sigma)$ are finite set of points, giving estimates of cardinality of these two sets at the same time. Note that $\Sigma \backslash f^{-1}(D)$ lies in the intersection of the zero divisors of $t_{1}, \cdots, t_{n-1}$ and $V$, and $V$ is clearly contained in a divisor determined by the sections $s_{0}, s_{1}, \cdots, s_{n}$ which define the mapping $F$. Applying Fatou's lemma to the Bézout estimate (3.1) in Proposition 3.2 we see that $\Sigma \backslash f^{-1}(D)$ consists of a finite number of points and the bound on the cardinality is independent of $\Sigma$. This gives an upper bound for $f(\Sigma) \backslash D$. To get an upper bound for $S \backslash f(\Sigma)$ one can pass to a normalization $\sigma: S^{\prime} \rightarrow S$ and prove the corresponding statement for $S^{\prime}$ and the Riemann surface $D^{\prime}=\sigma^{-1}(f(\Sigma))$, which is equipped with the induced ( possible degenerate) Hermitian metric $\left(f^{-1} \circ \sigma\right)^{*}\left(\left.\omega\right|_{\Sigma}\right)$. The degeneracies occur at some points corresponding to singularities of $\Sigma$. Such singularities can either come from singularities of $D$ itself or from the intersection of $\Sigma$ 
with $V$. The first set is finite since $Z$ is algebraic, and the second set is also finite by the Bézout estimate (3.1) in Proposition 3.2. Thus $\left(f^{-1} \circ \sigma\right)^{*}\left(\left.\omega\right|_{\Sigma}\right)$ is degenerate at at most a finite set of points $\left\{p_{i}\right\}_{i=1}^{r}$. Modify the metric in small compact neighborhoods of these points to get a smooth metric $\mu$. Then the Gauss-Bonnet integrals of these two metrics are related by

$$
\int_{D^{\prime}} c_{1}\left(D^{\prime}, \mu\right)=\int_{D^{\prime} \backslash\left\{p_{i}\right\}} c_{1}\left(D^{\prime},\left(f^{-1} \circ \sigma\right)^{*}\left(\left.\omega\right|_{\Sigma}\right)\right)-r
$$

where $c_{1}$ is denoted by the associated Chern form. Recall that the estimate (3.21) in Proposition 3.5 says

$$
\int_{D^{\prime} \backslash\left\{p_{i}\right\}} c_{1}\left(D^{\prime},\left(f^{-1} \circ \sigma\right)^{*}\left(\left.\omega\right|_{\Sigma}\right)\right) \geq-C q^{2 n-2} \int_{M} R i c^{n}
$$

which implies that

$$
\int_{D^{\prime}} c_{1}\left(D^{\prime}, \mu\right)>-C
$$

for a constant $C$ independent of the choice of hyperplane sections. By the well-known result of Huber [12] we have

$$
2 \operatorname{genus}\left(S^{\prime}\right)+\operatorname{card}\left(S^{\prime} \backslash D^{\prime}\right)-2 \leq-\frac{1}{2 \pi} \int_{D^{\prime}} c_{1}\left(D^{\prime}, \mu\right)<C .
$$

This gives the upper bound for the number of points in $S^{\prime} \backslash D^{\prime}$ and hence of $S \backslash f(\Sigma)$. Thus we obtain the uniform upper bound for $S \backslash D$. Therefore $F(M \backslash V)$ is Zariski open in $Z$.

Now we are in position to desingularize $F$ to a biholomorphism from $M$ to a quasi-projective variety. Recall that for any fixed point $x_{0} \in V$, one can choose $n+1$ holomorphic plurianticanonical sections $s_{0}^{\prime}, s_{1}^{\prime}, \cdots, s_{n}^{\prime} \in \Gamma^{2}\left(M, K^{-q^{\prime}}\right)$ which give the holomorphic coordinate at $x_{0}$. Although the integer $q^{\prime}$ may be different from the integer $q$ in the mapping $F$, we can add the sections $s_{0}^{\prime}, s_{1}^{\prime}, \cdots, s_{n}^{\prime}$ to $F$ to get a birational map $F^{\prime}$ by composing the sections and $F$ with a Veronese map as done in [17], [26]. Denote by $V^{\prime}$ the union of the branching locus and the base locus of $F^{\prime}$. Then $V^{\prime} \nRightarrow V$. If for each such a 
birational map, the union of the branching locus and the base locus has only finite number of irreducible components of maximal dimension, then it is clear that the birational map $F$ will become a biholomorphism after adding a finite number of holomorphic plurianticanonical sections in $\bigcup_{q>0} \Gamma^{2}\left(M, K^{-q}\right)$.

Recall a result of Demailly [5] which states that if $\operatorname{dim} V \leq p$, then the relative cohomology group $H^{2(n-p)}(M, M \backslash V, \mathbf{R})$ is isomorphic to $\mathbf{R}^{J}$, where $J$ is the number of irreducible components of dimension $p$ in $V$. As a consequence, if $H^{2(n-p)}(M, \mathbf{R})$ and $H^{2(n-p)-1}(M \backslash V, \mathbf{R})$ are finite dimensional, then the number of irreducible branches of $V$ of complex dimension $p$ is finite. In our case, $M$ is diffeomorphic to $\mathbf{R}^{2 n}$ and $M \backslash V$ is Zariski open in an algebraic variety. So $M \backslash V$ is of finite topological type. Hence we can desingularize the map $F$ into a biholomorphism from $M$ to a quasi-projective variety.This proves the first assertion of the Main Theorem.

To prove the second assertion of the Main Theorem we recall a theorem of Ramanujam [19] which states that a quasi-projective variety homeomorphic to $\mathbf{R}^{4}$ is biregular to $\mathbf{C}^{2}$. Thus $M$ is biholomorphic to $\mathbf{C}^{2}$ when the complex dimension $n=2$.

Therefore the proof of the Main Theorem is completed.

\section{References}

[1] Andreotti, A. and Vesentini, E., Carleman estimates for the LaplaceBeltrami operator on complex manifolds, Publ. Math. Inst. Hantes Études Sci., 25 (1965), 81-130.

[2] Burns, D., Shnider, S. and Wells, R. O., On deformations of strictly pseudoconvex domain, Invent. Math., 46 (1978), 237-253.

[3] Cheeger, J. and Gromoll, D., On the structure of complete manifolds of non-negative curvature, Ann. of Math., 46 (1972), 413-433. 
[4] Chen, B. L., Tang, S. H. and Zhu, X. P., A uniformization theorem of complete noncompact Kähler surfaces with positive bisectional curvature, Preprint.

[5] Demailly, J. P., Mesures de Monge-Ampére et caractérisation géometri que des variétés algébriques affines, Mem. Soc. Math. France, No.19(1985), 123 pages.

[6] Demailly, J. P., Holomorphic Morse inequalities, Proceedings of Symposia in Pure Mathematics, 52 (1991), Part 2.

[7] Fefferman, C., The Bergman kernel and biholomorphic mappings of pseudoconvex domains, Invent. Math., 26 (1974), 1-65.

[8] Greene, R. E. and Wu, H., $C^{\infty}$ convex functions and manifolds of positive curvature, Acta. Math., 137 (1976), 209-245.

[9] Greene, R. E., and Wu, H., Analysis on non-compact Kähler manifolds, Proc. Symp. Pure. Math. Vol 30, Part II, Amer. Math. Soc., (1977).

[10] Gromoll, D. and Meyer, W., On complete open manifolds of positive curvature, Ann. of Math., 90(1969), 75-90.

[11] Hörmander, L., $L^{2}$-estimates and existence theorems for the $\bar{\partial}$-operator, Acta. Math., 113 (1965), 89-152.

[12] Huber, A., On subharmonic functions and differential geometry in the large, Comm. Math. Helv., 32 (1957), 13-72.

[13] Li, P. and Schoen, R, $L^{p}$ and mean value properties of subharmonic functions on Riemannian manifolds, Acta Math. 153 (1984), 279-301. 
[14] Mok, N., An embedding theorem of complete Kähler manifolds of positive bisectional curvature onto affine algebraic varieties, Bull. Soc. Math. France, 112 (1984), 197-258.

[15] Mok, N., Compactification of complete Kähler surfaces of finite volume satisfying certain curvature conditions, Ann. of Math., 129 (1989), 383425 .

[16] Mok, N., An embedding theorem of complete Kähler manifolds of positive Ricci curvature onto quasi-projective varieties, Math. Ann., 286 (1990), 377-408.

[17] Mok, N. and Zhong J. Q., Compactifying complete Kähler-Einstein manifolds of finite topological type and bounded curvature, Ann. of Math., 129 (1989), 427-470.

[18] Nadel, A. and Tsuji, H., Compactification of complete Kähler manifolds of negative Ricci curvature, J. Differential Geometry, 28 (1988), 503-512.

[19] Ramanujam, C. P., A topological charaterization of the affine plane as an algebraic variety, Ann. of Math., 94 (1971), 69-88.

[20] Schoen, R. and Yau, S. T., Lectures on differential geometry, in Conference Proceedings and Lecture Notes in Geometry and Topology, Volume 1, International Press Publications, 1994.

[21] Shi, W. X., Ricci flow and the uniformization on complete noncompact Kähler manifolds, J. Diff. Geom., 45 (1997), 94-220.

[22] Simha, R. R., On the analyticity of certain singularity sets, J. Indian Math. Soc., 39(1975), 281-283.

[23] Siu, Y. T., Pseudoconvexity and the problem of Levi, Bull. Amer. Math. Soc., 84 (1978), 481-512. 
[24] Siu, Y. T. and Yau, S. T., Complete Kähler manifolds with non-positive curvature of faster than quadratic decay, Ann. of Math., 105 (1977), 225264.

[25] Tian, G., On a set of polarized Kähler metrics on algebraic manifolds, J. Differential Geometry, 32 (1990), 99-130.

[26] To, W. K., Quasi-projective embeddings of noncompact complete Kähler manifolds of positive Ricci curvature and satisfying certain topological conditions, Duke Math. J., 63 (1991), no. 3, 745-789.

[27] Yau, S. T., Problem Section, Seminar on Diff. Geom. edit by S. T. Yau, Princeton Univ. Press, 1982.

[28] Yau, S. T., A review of complex differential geometry, Proc. Symp. Pure Math., Vol 52, Part II, Amer. Math. Soc., (1991). 